OPEN ACCESS

Edited by:

Xia Li,

Shenzhen Institutes of Advanced Technology (CAS), China

Reviewed by:

Mingwei Chen,

The First Affiliated Hospital of

Soochow University, China

Edmund Ui-Hang Sim,

Universiti Malaysia Sarawak, Malaysia

Kevin Tak-Pan Ng,

The University of Hong Kong,

Hong Kong

*Correspondence:

Manyi Yang

yangmanyi@csu.edu.cn

${ }^{t}$ These authors have contributed equally to this work

Specialty section:

This article was submitted to Gastrointestinal Cancers,

a section of the journal

Frontiers in Oncology

Received: 04 February 2021

Accepted: 22 June 2021

Published: 13 July 2021

Citation:

Li B, Zhou M, Wang J, $\mathrm{Xu} \mathrm{H}$ and Yang M (2021) Suppressing ERK Pathway Impairs Glycochenodeoxycholate-Mediated Survival and Drug-Resistance in Hepatocellular Carcinoma Cells.

Front. Oncol. 11:663944. doi: 10.3389/fonc.2021.663944

\section{Suppressing ERK Pathway Impairs Glycochenodeoxycholate-Mediated Survival and Drug-Resistance in Hepatocellular Carcinoma Cells}

\author{
Bingxin $\mathrm{Li}^{1+}$, Maojun Zhou ${ }^{2+}$, Jue Wang ${ }^{1}$, Hongjuan $\mathrm{Xu}^{1}$ and Manyi Yang ${ }^{1 *}$ \\ ${ }^{1}$ Department of Hepatobiliary and Pancreatic Surgery, National Health Commission (NHC) Key Laboratory of Nanobiological \\ Technology, Xiangya Hospital, Central South University, Changsha, China, ${ }^{2}$ Department of Oncology, NHC Key Laboratory \\ of Cancer Proteomics, National Center for Geriatrics Clinical Research, State Local Joint Engineering Laboratory for \\ Anticancer Drugs, Xiangya Hospital, Central South University, Changsha, China
}

Glycochenodeoxycholate (GCDA), a toxic component in bile salts, is involved in carcinogenesis of gastrointestinal tumors. The objective of this research was to study the function of ERK1/2 in the GCDA-mediated survival and drug-resistance in hepatocellular carcinoma cells (HCCs). Firstly, extracellular signal-regulated kinase 1/2 (ERK1/2) was detected extensively expressed in liver cancer cells, and silencing ERK $1 / 2$ by RNA interference could suppress GCDA-stimulated survival and promote apoptosis. Furthermore, phosphorylation of endogenous ERK $1 / 2$ could be potently stimulated by GCDA in combination with enhanced chemoresistance in QGY-7703 hepatocellular carcinoma cells. The GCDA-mediated proliferation and chemoresistance could be impaired by PD98059, which acted as an inhibitor to block the phosphorylation of ERK1/2. Mechanistically, PD98059 was able to potently suppress GCDA-stimulated nuclear aggregation of ERK $1 / 2$ and $\mathrm{p}-\mathrm{ERK} 1 / 2$, upregulate pro-survival protein Mcl-1 and downregulate pro-apoptotic protein Bim. The results of this study indicated that disruption of ERK1/2 by blocking phosphorylation or nuclear translocation may put forward new methods for solving the problem of GCDA-related proliferation and drug-resistance in liver cancer treatment.

Keywords: hepatocellular carcinoma cells, glycochenodeoxycholate, extracellular signal-regulated kinase 1/2, anti-apoptosis proteins, pro-apoptotic proteins

\section{INTRODUCTION}

Hepatocellular carcinoma (HCC) is the most common liver cancer nowadays, and more than 700,000 cases are diagnosed every year (1). The pathogenesis of HCC is extremely complex, but evolving information suggests that the major risk factors for HCC in contemporary clinical practice include alcoholism, non-alcoholic fatty liver disease (NFLD), hepatitis B virus (HBV), and hepatitis $\mathrm{C}$ virus (HCV) $(2,3)$. Most patients with advanced liver cancer will choose chemotherapy. However, patients with HCC usually develop resistance to 5 -fluorouracil, doxorubicin or cisplatin, which are the traditional chemotherapeutics. Unfortunately, sorafenib, the new generation of drugs, did not 
achieve the desired results (4). Thus, it is very important to explore the resistance mechanism of HCC.

Bile salts are the major ingredients in bile, which are secreted by liver cells and involved in fat digestion and absorption. Glycochenodeoxycholate (glycine conjugate of chenodeoxycholate, GCDA), a toxic component in bile salts, is involved in carcinogenesis of gastrointestinal tumors (5). Previous researches have indicated that GCDA could stimulate the growth of Barrett's adenocarcinoma cells and non-neoplastic Barrett cell lines through PI3 kinase/Akt pathway and p38/ERK/MAPK pathway respectively $(6,7)$. Satoshi et al. (8) found that glycochenodeoxycholate acid could promote the proliferation of intestinal epithelia via decreasing cyclic AMP and increasing histone $\mathrm{H} 2 \mathrm{AX}$ phosphorylation after exposure to $\gamma$-rays. Another study demonstrated that the biliary tract cancer could be induced by GCDA via aggregation of 8-OHdG and oxidative DNA damage (9). The metabolic disorder of bile salts could lead to abnormal bile salt accumulation; it could be a direct factor in the development of HCC. A study by Wang et al. (10) found that GCDA might upregulate pro-survival proteins (Mcl-1, Survivin, and Bcl-2) and eventually results in chemoresistance of HCC cells. However, the specific intracellular mechanism of GCDA-mediated hepatocellular carcinoma development remains to be further studied.

As a member of the mitogen activated protein kinase family, the extracellular signal-regulated kinase (ERK) takes a key part in transmitting signals from receptors on the cell surface into the nucleus (11). Signals transmitted from MEK1/2 can phosphorylate ERK1/2 at Thr and Tyr residues (12). Then the activated ERK1/2 phosphorylates downstream substrates and eventually causes cell proliferation, differentiation, and canceration (13). Usually, ERK1/2 is mainly distributed in the cytoplasm of normal cells. Upon stimulation, many ERK1/2 molecules shift to the nucleus, Golgi, mitochondria, endosomes/lysosomes and endoplasmic reticulum (14). The main translocation seems to be the entry into the nucleus, which is an important place for signal transmission downstream of ERK (13). Because the nuclear translocation of ERK is mainly important for cell proliferation, prevention of such translocation can be used as a novel strategy to combat cancer (15). Furthermore, ERK1/2 signaling is an important regulator of cell-intrinsic Bcl-2-regulated apoptotic signaling (16). In most situations, ERK1/2 signaling accelerates cell growth via stimulating anti-apoptosis proteins (Bcl-2, Mcl-1, and $\mathrm{Bcl}-\mathrm{xL}$ ) and inhibiting pro-apoptotic proteins (Bim, Bad, Bmf, and Puma) (14). Thus, suppression of ERK1/2 pathway in tumor cells might serve as an effective way to prevent cancer development.

The chemoresistance of ERK1/2 has been extensively studied in other cancers. In radioresistant glioblastoma multiforme cells, cell survival could be promoted through ERK1/2 signaling when pSTAT3(Y705) was inhibited (17). ERK1/2 and p38 MAPK signaling pathways were significantly involved in neoplastic transformation and cisplatin-resistance in nasopharyngeal carcinoma cell lines (18). However, there was little in-depth research for the chemoresistance of ERK1/2 in HCC. A published study has shown that the activation of ERK1/2 could decrease the sensitivity to sorafenib in the HCC cells (Bel-7402 and SMMC-7721) (19). Our previous studies have confirmed the association of GCDA with drug resistance in HCC cells $(10,20)$. But the exact function of ERK1/2 in such process has not been clarified. In this research, we proved that GCDA mediates activation and nuclear accumulation of ERK1/2, which finally results in promoting anti-apoptotic function in human liver cancer cells.

\section{MATERIALS AND METHODS}

\section{Cell Culture}

LO2, HepG2, Bel-7402, Bel-7404, SMMC-7721, Huh7, MHC97$\mathrm{H}$, and QGY-7703 HCC cell lines were originally from the Institute of Biochemistry and Cell Biology (CAS, Shanghai, China). LO2 and Bel-7402 cell line were maintained in RPMI1640 medium (Thermo Fisher Scientific, Waltham, USA) with 10\% fetal bovine serum (ExCell Bio, Shanghai, China). HepG2, Bel-7404, SMMC-7721, Huh7, MHC97-H, and SMMC-7721 QGY-7703 cell lines were cultivated in Dulbecco's modified Eagle's medium (Hyclone, Logan, USA) supplemented 10\% FBS. Cell lines were incubated at $37^{\circ} \mathrm{C}$ with $5 \% \mathrm{CO}_{2}$.

\section{Reagents and Antibodies}

The antibodies of ERK1 + ERK2 and ERK1 (pT202/pY204) + ERK2 (pT185/pT187) were obtained from Abcam (Cambridge, UK). Goat-anti rabbit HRP antibody and anti-GAPDH antibody were from Cell Signaling Technology (Danvers, MA, USA). PD98059, a specific inhibitor of ERK kinase, was from Calbiochem (San Diego, CA, USA). Glycochenodeoxycholate (GCDA) and cisplatin were obtained from Sigma-Aldrich (St. Louis, USA). 5-Fluorouracil (5-FU) was purchased from Xudong Haipu Pharmaceutical (Shanghai, China). The Annexin V-FITC apoptosis detection kit was purchased from Becton, Dickinson and Company (BD, Franklin Lake, NJ).

\section{siRNA and Transfections}

For RNA interference, siRNA 225 (ACACGCAGUU GCAGUACAU), 888 (GACCGGAUGUUAACCUUUA), and 933 (GAAACUACCUACAGUCUCU) targeting human ERK1, siRNA 355 (GUGCUCUGCUUAUGAUAAU), 513 (CACCAACCAUCGAGCAAAU) and 714 (CCACC UGUGAUCUCAAGAU) targeting human ERK2 and negative control siRNA (UUCUCCGAACGUGUCACGU) were from Shanghai Gene Pharma, Co., Ltd (Shanghai, China). QGY-7703 cells were transfected with siRNAs for $24 \mathrm{~h}$ using Lipofectamine RNAi max (Invitrogen, NY, USA).

\section{CCK8 Assay}

QGY-7703 cells were seeded in 96 well plates. Then GCDA, drugs, or inhibitors were used to treat cells. After various treatments, each well was supplemented with $10 \mu \mathrm{l}$ of CCK8 solution and incubated for $1.5 \mathrm{~h}$. After that, the absorbance was determined by microplate microscopy at $450 \mathrm{~nm}$ (BioTek, Winooski, VT). 


\section{Western Blot Analysis}

The samples of QGY-7703 cells were lysed with detergent buffer for $30 \mathrm{~min}$ on ice. Then cell products were scraped from the wells and centrifuged for $15 \mathrm{~min}$ at $12,000 \mathrm{rpm}$. Protein, $30 \mu \mathrm{g}$, was loaded onto $10 \%$ SDS-PAGE and transferred to a polyvinylidene difluoride (PVDF) membrane. After blocking with blocking solution for $2 \mathrm{~h}$ at room temperature, cells were then incubated at $4^{\circ} \mathrm{C}$ overnight with primary antibodies, followed by washing with $1 \times$ TBST and incubating with horseradish peroxidase-conjugated anti-mouse or anti-rabbit secondary antibodies $(1: 5,000)$ with shaking for $1 \mathrm{~h}$. Results were detected using WesternBright ${ }^{\mathrm{TM}}$ ECL (Advansta, USA), and the bands were scanned and quantified using the FluorChem FC3 system.

\section{Flow Cytometry}

QGY-7703 cells were transfected with siRNA888 and siRNA513 together for $24 \mathrm{~h}$. Following treatment with $100 \mu \mathrm{M}$ GCDA, cells were collected and washed with cold PBS. After resuspending with $1 \times$ binding buffer, $3 \mu \mathrm{l}$ Annexin V-FITC and propidium iodide (PI) (Becton, Dickinson and Company, NJ) were used to treat the cells for $15 \mathrm{~min}$. The apoptotic rate was detected by flow cytometry.

\section{Immunofluorescence}

In 24-well plates, QGY-7703 cells were cultured with a glass coverslip overnight. After cells were exposed to GCDA or
GCDA + PD98059 for 8 h, 4\% paraformaldehyde was used to fix cells for $15 \mathrm{~min}$. The cells were washed with TBST and performed using ERK1/2 or p-ERK1/2 antibody at $4^{\circ} \mathrm{C}$ overnight after incubating with Alexa Fluor ${ }^{\circledR} 594$ goat antibody at $37^{\circ} \mathrm{C}$ for $1 \mathrm{~h}$. Cell nuclei were stained with DAPI for $2 \mathrm{~min}$. At last, the results were photographed with a fluorescence microscope.

\section{Statistical Analysis}

SPSS software V17.0 was used to perform the statistical analysis. All data were displayed as the means \pm SD. Inter-group differences were assessed by Student's t-test. $\mathrm{P}<0.05$ was the considered level of statistical significance.

\section{RESULTS}

\section{ERK1/2 Acts a Part in GCDA-Induced Survival of Human Liver Carcinoma Cells}

The ERK1/2 cascade is best known for its role in proliferation, differentiation, and tumorigenesis (13). Firstly, we measured the endogenous protein levels of ERK1/2 in normal liver cells (LO2) and seven HCC cell lines (HepG2, Bel-7402, Bel-7404, SMMC7721, Huh7, MHC97-H, and QGY-7703. The result of Western blot showed that ERK1/2 was extensively expressed in all the liver cancer cells we detected (Figure 1A). Next, to test whether GCDA promoted HCC cell proliferation, we treated

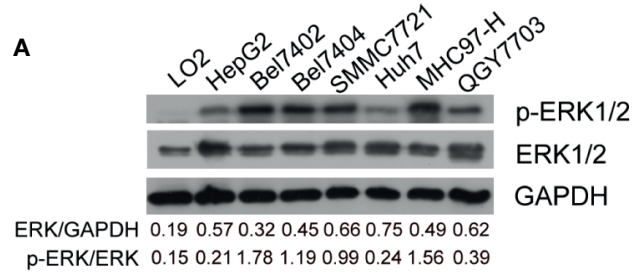

C

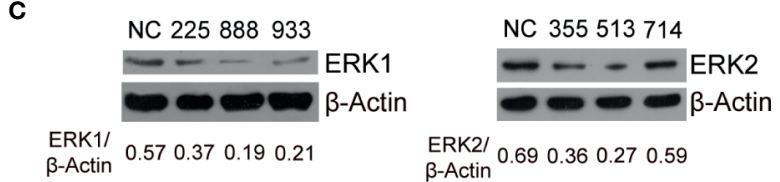

D

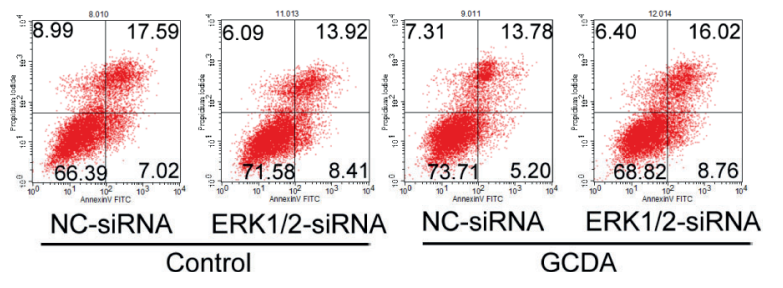

B

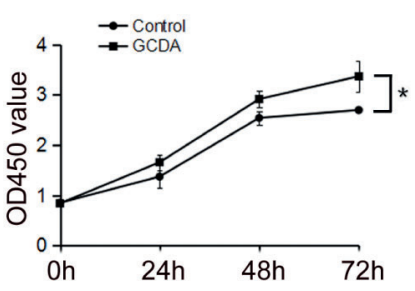

E

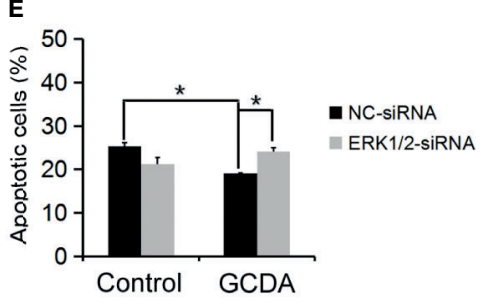

FIGURE 1 | ERK1/2 act a part in GCDA-mediated survival of human liver carcinoma cells. (A) Expression of ERK1/2 in normal liver cells (LO2) and seven liver carcinomas cell lines (HepG2, Bel7402, Bel7404, SMMC7721, Huh7, MHC97-H and QGY7703) was detected by western blot and quantified by Alphaview software.

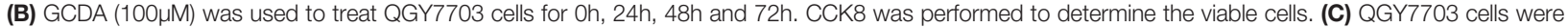
transfected with siRNA targeting ERK1 (225, 888 and 933) and ERK2 (355, 513 and 714). After 24h, whole cell extracts were analyzed by western blot using ERK1 and ERK2 antibodies. NC, negative; control siRNA. (D, E) siRNA888 targeting ERK1 and siRNA513 targeting ERK2 were transfected into QGY7703 cells together. 24 hours later, $100 \mu \mathrm{M}$ GCDA was used to treat cells for $24 \mathrm{~h}$. Apoptosis were determined using flow cytometry. All data represent the mean \pm SD and were obtained from at least three independent experiments. ${ }^{*} \mathrm{P}<0.05$, (Student's t-test). 
QGY-7703 cell line with $100 \mu \mathrm{M}$ GCDA for $0,24,48$, and $72 \mathrm{~h}$, and then checked the viable cells by CCK8. Results indicated that viable cells significantly increased after treatment with GCDA for72 h (Figure 1B).

To determine whether ERK1/2 affected the GCDA-induced survival of HCC cells, we designed siRNAs targeting ERK1 (225, 888, and 933) and ERK2 $(355,513$, and 714). All the siRNAs were transfected into QGY-7703 cells. Then immunoblotting was done to determine the interference efficiency. As shown in Figure 1C, ERK1 and ERK2 protein expressions were inhibited by siRNA888 and siRNA513, respectively. After siRNA888 targeting, ERK1 and siRNA513 targeting, ERK2 was transfected into QGY-7703 cell line together; GCDA was used to treat the cells for $24 \mathrm{~h}$. Apoptotic cells were analyzed using annexin $\mathrm{V}$ binding on FASC. Flow cytometry results demonstrated that GCDA could repress apoptosis. But after ERK1/2 was silenced, the apoptotic cells were increased (Figures 1D, E). In other words, specific depletion of ERK1/2 blocked GCDA-stimulated cell survival. These results indicated that ERK1 and ERK2 molecules have played a role in the survival of hepatoma cells mediated by GCDA.

\section{GCDA Induces ERK1/2 Phosphorylation, Which May Be Involved in Prolonged Survival of Human Liver Cancer Cells}

Furthermore, we investigated potential mechanisms involved in the GCDA-induced HCC cell survival. QGY-7703 and Huh7 cells were treated with $100 \mu \mathrm{M}$ GCDA for $0,0.5,1,2,4,8,12$, and
$24 \mathrm{~h}$. Results demonstrated that the activated ERK1/2 increased obviously after GCDA treatment in QGY-7703 and Huh7 cells, while the expression of endogenous ERK1/2 changed little (Figures 2A, B).

Cisplatin has been known as one of the most potential and widely used drugs, which is effective in a variety of solid cancers such as testicular, ovarian, head and neck, bladder, lung, cervical, melanoma, and lymphomas (21-25). The antimetabolite 5fluorouracil (5-FU), which can inhibit thymidylate synthase, is a widely used antitumor agent (26). In order to check the effect of GCDA-induced ERK1/2 activation on cell survival, QGY-7703 and Huh7 cells were treated with antitumor drug (cisplatin or 5FU) or GCDA $(100 \mu \mathrm{M})$ + antitumor drug (cisplatin or 5-FU) for $72 \mathrm{~h}$. The IC50 of cisplatin for QGY-7703 is $8.8 \mu \mathrm{M}$ (Figure 2C). The IC50 value of 5-FU is $0.9 \mu \mathrm{g} / \mathrm{ml}$ for QGY-7703 and $2.7 \mu \mathrm{g} / \mathrm{ml}$ for Huh7, respectively (Figures 2D, E). However, following GCDA treatment, the IC50 concentrations were increased obviously. Such results indicate that GCDA can significantly enhance resistance to drugs. Therefore, we speculated the involvement of activated ERK1/2 in chemoresistance induced by GCDA.

\section{The MAPK/ERK1/2 Inhibitor PD98059 Decreases GCDA-Stimulated Cell Proliferation}

To further verify the role of activated ERK1/2 in HCC cells, the MAPK/ERK1/2 inhibitor PD98059, which could inhibit phosphorylation of ERK1/2, was used (27). We treated QGY-
A

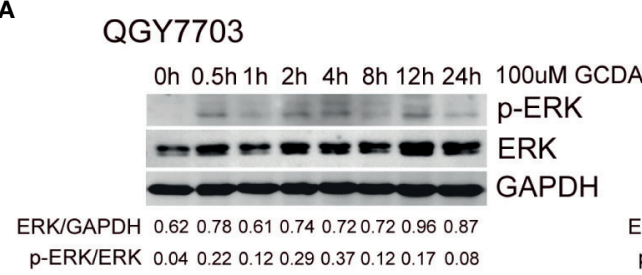

B

Huh7

Oh $0.5 \mathrm{~h} 1 \mathrm{~h} 2 \mathrm{~h} 4 \mathrm{~h} 8 \mathrm{~h} 12 \mathrm{~h} 24 \mathrm{~h} 100 \mathrm{uM}$ GCDA

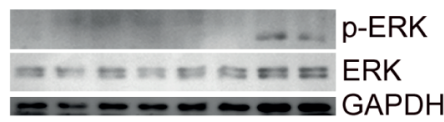

ERK/GAPDH 0.550 .550 .530 .370 .440 .460 .620 .72 p-ERK/ERK $0.190 .450 .690 .610 .200 .150 .66 \quad 0.34$
C

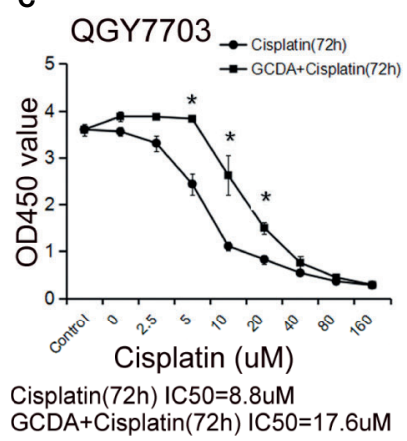

D

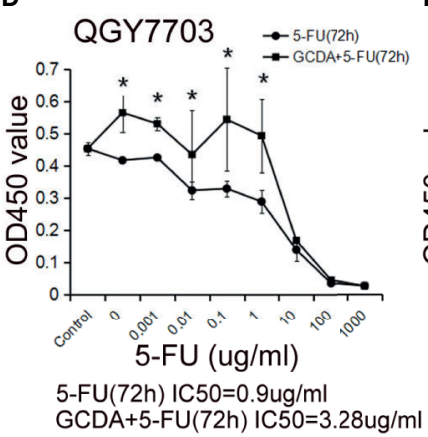

E

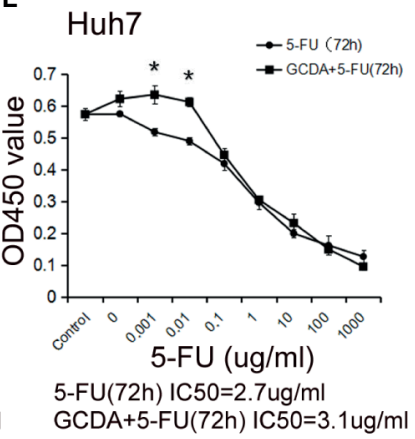

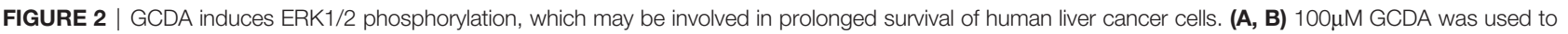
treat QGY7703 and Huh7 cells for Oh, 0.5h, 1h, 2h, 4h, 8h, 12h and 24h. The expression level of ERK1/2 and p-ERK1/2 were tested by western blot and quantified

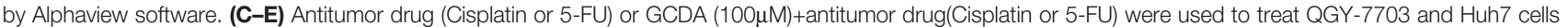
for $72 \mathrm{~h}$. CCK8 was performed to determine the viable cells. IC50 is calculated as the concentration of Cisplatin or 5 -FU inducing a 50\% reduction in cell viability. All data represent the mean $\pm \mathrm{SD}$ and were obtained from at least three independent experiments. ${ }^{*} \mathrm{P}<0.05$ (Student's t-test). 
7703 cells with GCDA $(100 \mu \mathrm{M})$ or GCDA $(100 \mu \mathrm{M})+$ PD98059 $(10 \mu \mathrm{M})$ for 24,48 , and $72 \mathrm{~h}$. Then, CCK8 was done to test the viability of QGY-7703 cells. CCK8 experiments showed that suppression of ERK1/2 activation by PD98059 would decrease proliferation of liver cancer cells (Figure 3A). Next, QGY-7703 cells were treated with or without PD98059 $(10 \mu \mathrm{M})$ for $0.5 \mathrm{~h}$, followed by treatment with GCDA $(100 \mu \mathrm{M})$ or GCDA $(100 \mu \mathrm{M})+$ antitumor drug $(1 \mu \mathrm{g} / \mathrm{ml} 5-\mathrm{FU})$ for $72 \mathrm{~h}$. Results of CCK8 showed that PD98059 significantly attenuated the chemoresistance induced by GCDA, which could prolong cell survival following treatment with 5-FU (Figure 3B). In conclusion, these findings implied that phosphorylation (or activation) of ERK1/2, which is attenuated by PD98059, is important for the survival and chemoresistance of GCDAmediated HCC cells.

\section{PD98059 Suppresses GCDA-Induced Nuclear Aggregation of ERK1/2 and p-ERK1/2}

In unstimulated cells, ERK1/2 molecules are usually located in the cytoplasm (15). Under stimulation, numerous ERK1/2 molecules are translocated to the nucleus (15). ERK1/2 localization plays a significant role in determining the strength of this pathway. Therefore, we examined the localization of ERK1/2 and p-ERK1/2 following GCDA $(100 \mu \mathrm{M})$ or GCDA $(100 \mu \mathrm{M})+$ PD98059 $(10 \mu \mathrm{M})$ treatment. The results of immunofluorescence staining showed that ERK1/2 proteins were distributed in both cytoplasm and nucleus and more pERK1/2 proteins accumulated in the nucleus as small spots in resting HCC cells (Figures $\mathbf{4 A}, \mathbf{B}$ ). Following GCDA treatment,

A

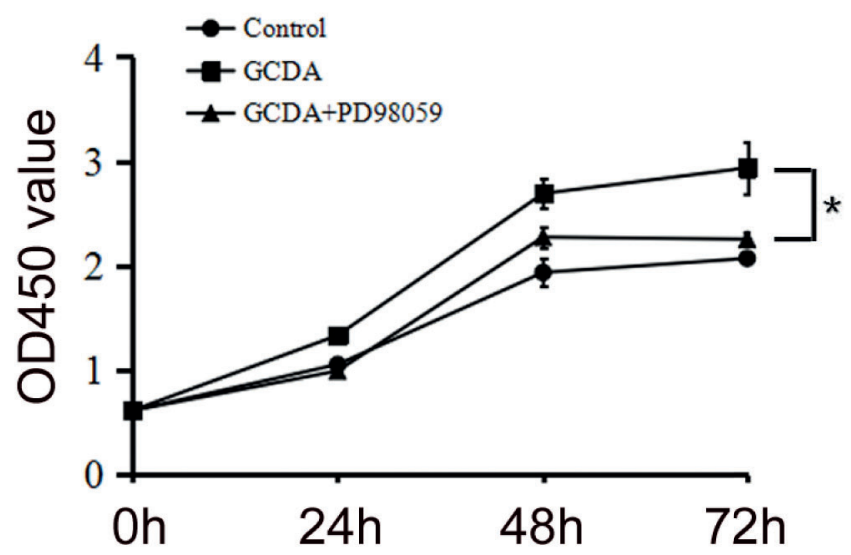

B

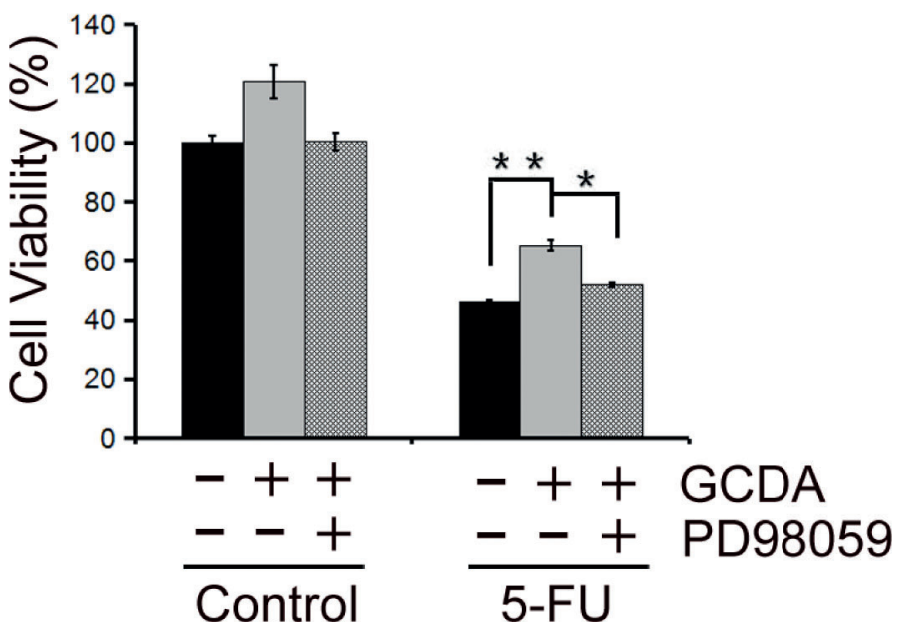

FIGURE 3 | PD98059, the ERK1/2 inhibitor, attenuates GCDA-mediated survival and drug-resistance in HCC cells. (A) PD98059 could inhibit phosphorylation of

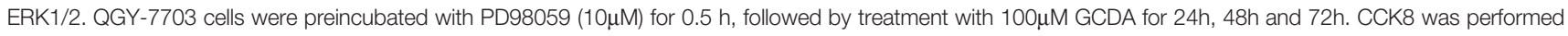

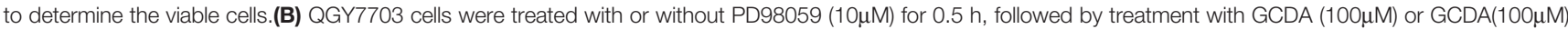
+ antitumor drug $(1 \mu \mathrm{g} / \mathrm{ml} 5$-FU) for $72 \mathrm{~h}$. Then CCK8 was performed to determine the viable cells. Data in graphs are as mean $\pm \mathrm{SD}$. All experiments data were repeated at least three independent experiments. ${ }^{*} \mathrm{P}<0.05,{ }^{* *} \mathrm{P}<0.01$ (Student's t-test). 


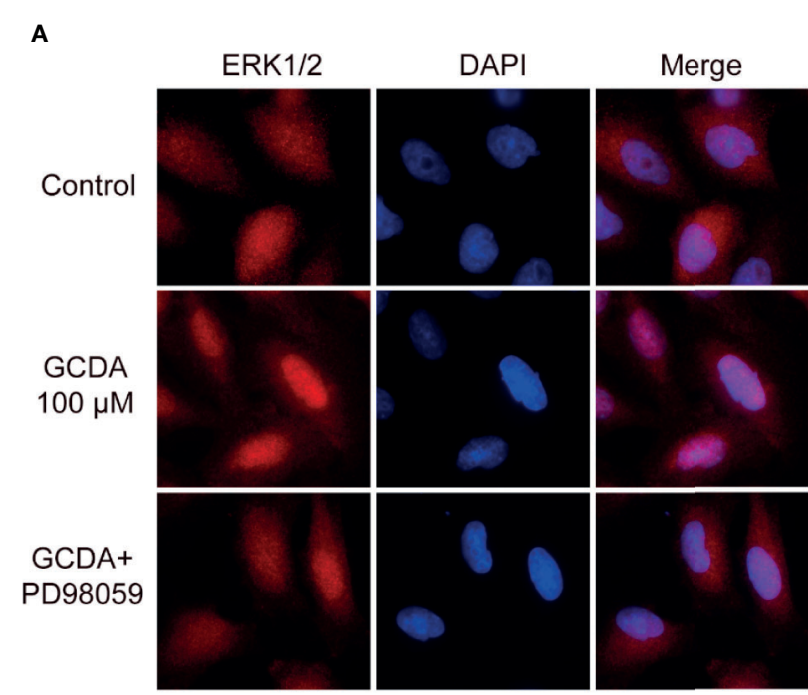

B

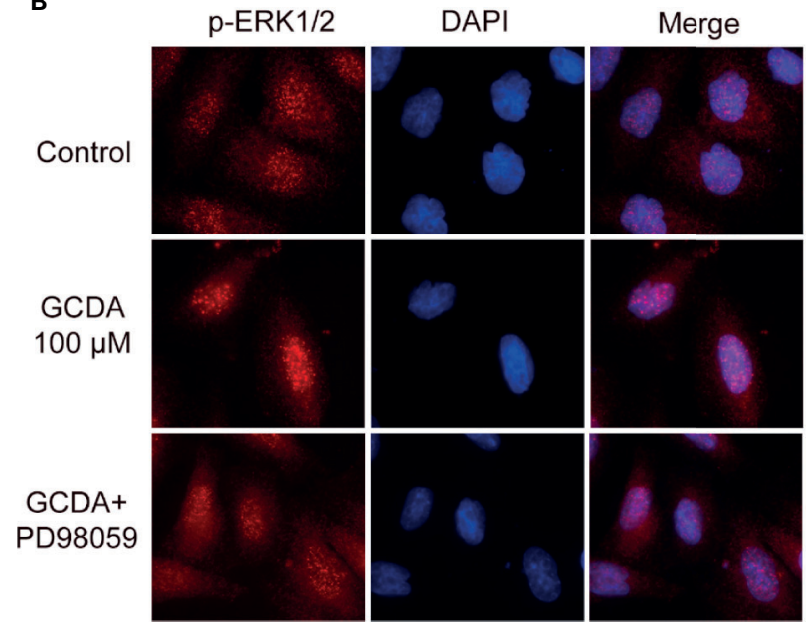

FIGURE 4 | PD98059 suppresses GCDA-induced nuclear aggregation of ERK1/2 and p-ERK1/2. (A, B) QGY-7703 cells were preincubated with PD98059 $(10 \mu \mathrm{M})$ for $0.5 \mathrm{~h}$, followed by treatment with $100 \mu \mathrm{M}$ GCDA for $8 \mathrm{~h}$. Immunofluorescence staining was done using ERK $1 / 2$ or p-ERK $1 / 2$ antibody. Cell nuclei were stained with DAPI for 2 min. The experiments were repeated three times.

most ERK1/2 proteins gathered in the nucleus, while more $\mathrm{p}$ ERK1/2 proteins accumulated in the nucleus as bigger speckles. However, after PD98059 treatment, the aggregation of ERK1/2 and $\mathrm{p}$-ERK1/2 proteins in the nucleus significantly decreased (Figures 4A, B). Collectively, the above data suggested that nuclear accumulation of ERK1/2 and p-ERK1/2 induced by GCDA could be impaired by PD98059.

\section{PD98059 Restrains GCDA-Induced Increase of $\mathrm{Mcl}-1$ and Decrease of Bim}

ERK1/2 signaling has been verified to have the ability to regulate some members of the Bcl-2 family, which can contribute to tumor cell survival via increasing anti-apoptotic factors and decreasing pro-apoptotic members of Bcl-2 family (16). Hence, we inspected the level of some Bcl-2 family members following GCDA (which can activate ERK1/2 pathway) or PD98059 (which can repress ERK1/2 pathway) treatment. Firstly, $100 \mu \mathrm{M}$ GCDA was used to treat QGY-7703 cells for $0,0.5,1$, 2,4 , and $8 \mathrm{~h}$. Immunoblot had been done to check the levels of Bcl-2, Mcl-1, Bim, and Bak. We observed that GCDA could promote expression of Bcl-2 and Mcl-1, both of which are antiapoptotic Bcl-2 family members and decrease expression of Bim and Bak, both of which are pro-apoptotic Bcl-2 family members (Figure 5A). Next, in order to determine whether the suppression of ERK1/2 signaling regulated expression of $\mathrm{Bcl}-2$ family members, GCDA $(100 \mu \mathrm{M})$ or GCDA $(100 \mu \mathrm{M})+$ PD98059 $(10 \mu \mathrm{M})$ was used to treat QGY-7703 cells for $8 \mathrm{~h}$. Results showed that inhibition of ERK1/2 by PD98059 could block GCDA-induced increase of Mcl-1 and decrease of Bim. However, Bcl-2 and Bak did not change significantly (Figure 5B). Our data supported the notion that GCDA might facilitate cell survival via regulation proteins of Bcl-2 family, some of which could be inhibited by PD98059. Such results indicated that activation of ERK1/2 pathway induced by GCDA could mediate certain members of the Bcl-2 family.

\section{DISCUSSION}

Glycochenodeoxycholate is one of the toxic bile salts and may promote HCC invasion via activation of autophagy $(28,29)$. In the current study, survival and chemoresistance to cisplatin and 5-FU induced by GCDA have been verified in QGY-7703 cell line (Figures 1B and 2C-E).

The ERK1/2 signaling pathway is considered to have great effects on proliferation, invasion, and migration in cancer cells. Numerous studies have confirmed that ERK1/2 signaling is the main regulator that promotes the progression of human hepatocellular carcinoma (30-34). ERK1/2 participates in liver injury in human liver stem cells $(35,36)$. Also, the aggressive behavior of HCC cells has a positive relationship with the level of phosphorylated ERK and activated level of hepatic stellate cells (aHSCs) (37). Thus, we speculated GCDA mediated survival and chemoresistance via the ERK1/2 pathway in liver cancer cells. Our results showed that activation levels of ERK1/2 increased significantly following GCDA treatment in hepatocellular carcinoma cells (Figures 2A, B). After ERK1/2 was silenced by siRNA or phosphorylation of ERK1/2 was blocked by PD98059, cell proliferation was significantly decreased (Figures 1D, E and 3A). In the light of those results, it is reasonable to suggest that the ERK1/2 pathway is involved with GCDA-induced survival in HCC cells.

Because of binding to many scaffold proteins or cytoplasmic anchors in resting cells, ERK $1 / 2$ is usually localized in the cytoplasm (15). Upon stimulation, numerous ERK1/2 molecules are translocated to the nucleus (14). In QGY-7703 cells, ERK1/2 and p-ERK1/2 could aggregate in the nucleus after treatment with GCDA (Figures 4A, B). Therefore, nuclear aggregation of ERK1/2 molecules must be relevant to HCC cell 
A

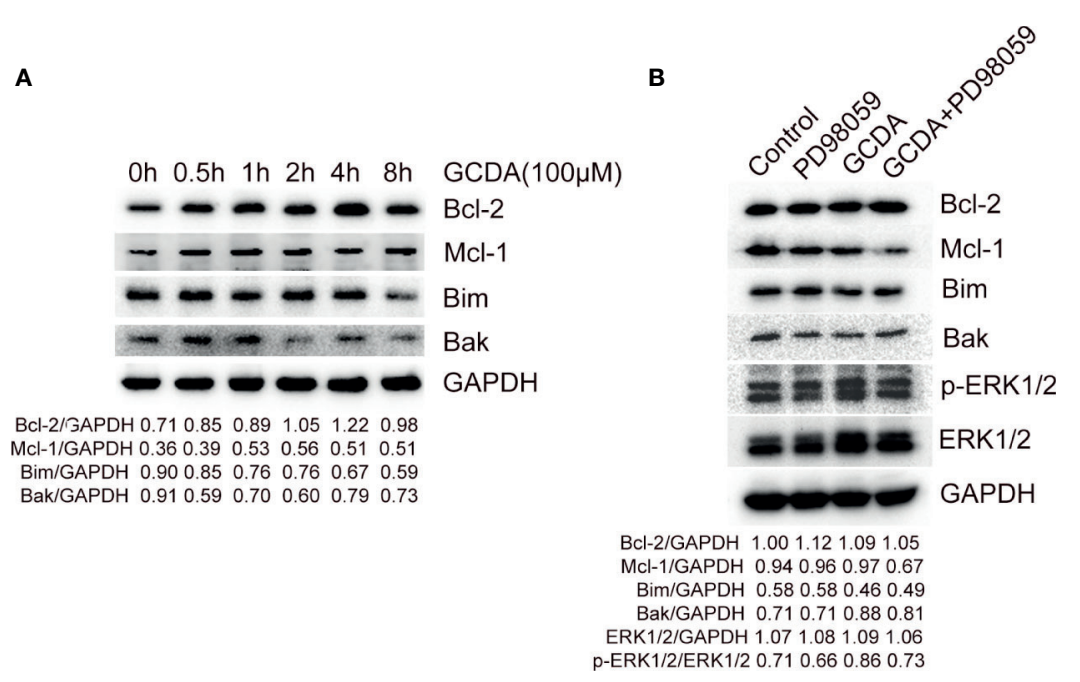

B

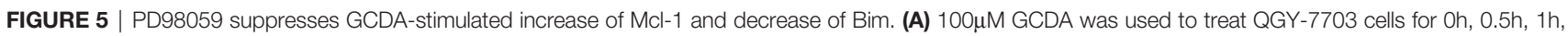
$2 \mathrm{~h}, 4 \mathrm{~h}$ and $8 \mathrm{~h}$. Expression of Bcl-2, Mcl-1, Bim and Bak was tested by western blot and quantified by Alphaview software. (B) QGY-7703 cells were preincubated

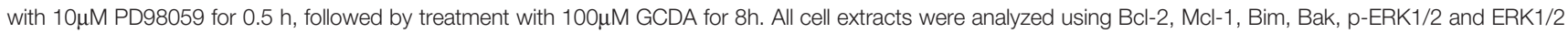
antibodies by Western blotting and quantified by Alphaview.

proliferation signal transduction following GCDA treatment. However, such nuclear accumulation could be decreased by inhibitor PD98059 (Figures 4A, B), which meant that the GCDA-induced survival signal is impaired by PD98059. Based on the evidence in this study, preventing ERK1/2 from entering the nucleus may be considered as a novel strategy to arrest liver cancer growth.

Activated ERK1/2 is also translocated to mitochondria, Golgi, the endoplasmic reticulum, or endosomes/lysosomes, thereby influencing cell physiology (38). Among them, the mitochondrial anchored ERK1/2 molecules are involved with the mitochondrial apoptosis pathway via affecting Bcl-2 family members (16). Usually, ERK1/2 signaling facilitates cell survival via activating pro-survival proteins (Bcl-2, Mcl-1, and Bcl-xL) and inhibiting pro-apoptotic proteins (Bim, Bad, Bmf, and Puma) (16). Among them, the transcription of pro-survival protein $\mathrm{Bcl}-2$ can be promoted by ERK1/2 signaling through cAMP-responsive element-binding protein (CREB) (39). Besides, Bcl-2 itself can also be phosphorylated at Ser87 by ERK1/2, which is proposed to inhibit its pro-survival function (14). The mRNA level of Mcl-1 is verified to be promoted in response to ERK1/2 pathway via CREB or transcription factor ELK1 (40). Also, the short half-life of Mcl-1 protein can be prolonged via direct phosphorylation by ERK1/2 (41). Bim, is a prominent target of ERK1/2 signaling (42). ERK1/2-induced activation of Bim leads to ubiquitylation and degradation (43). Bak is the apoptotic effector protein of Bcl2 family. Bak can be directly activated by Bim and cause the release of cytochrome c (44). In the present research, we observed that inhibiting ERK1/2 phosphorylation by PD98059 blocked GCDA-induced increase of Mcl-1 and decrease of Bim. However, Bcl-2 and Bak did not change significantly (Figures 5A, B). These results showed that the GCDA-induced change of Mcl-1 and Bim might be regulated by ERK1/2 pathway, while the variation of Bcl-2 and Bak may be induced by GCDA in an ERK-independent manner.

In conclusion, the present results found that GCDAstimulated cell proliferation and chemoresistance could be attenuated via targeting the ERK pathway. GCDA was able to potently promote phosphorylation and nuclear aggregation of ERK1/2 molecules, which eventually led to the increased level of anti-apoptotic Bcl-2 family member proteins (Bcl-2 and $\mathrm{Mcl}-1$ ) and the decreased expression of pro-apoptotic Bcl-2 family members (Bim and Bak). The inhibitor PD98059 not only suppressed the phosphorylation of ERK1/2, but also blocked ERK1/2 nuclear accumulation of the nucleus and attenuated GCDA-stimulated increase of Mcl-1 and decrease of Bim. Therefore, disruption of the pro-survival function of GCDA by blocking phosphorylation and nuclear accumulation of ERK1/2 molecules represents tactics for treating GCDA-related liver cancer and chemoresistance.

\section{DATA AVAILABILITY STATEMENT}

The original contributions presented in the study are included in the article/Supplementary Material. Further inquiries can be directed to the corresponding author.

\section{AUTHOR CONTRIBUTIONS}

MY contributed to conceive and designed the experiments. BL performed the data analyses and wrote the manuscript. MZ contributed significantly to analysis and manuscript preparation. JW and HX helped perform the analysis with constructive 
discussions. All authors contributed to the article and approved the submitted version.

\section{FUNDING}

This work was supported by the National Natural Science Foundation of China (grant numbers 81703412, 81402001)

\section{REFERENCES}

1. Bruix J, Gores GJ, Mazzaferro V. Hepatocellular Carcinoma: Clinical Frontiers and Perspectives. Gut (2014) 63(5):844-55. doi: 10.1136/gutjnl-2013-306627

2. Ioannou GN, Feld JJ. What Are the Benefits of a Sustained Virologic Response to Direct-Acting Antiviral Therapy for Hepatitis C Virus Infection? Gastroenterology (2019) 156(2):446-60.e2. doi: 10.1053/j.gastro.2018.10.033

3. Kulik L, El-Serag HB. Epidemiology and Management of Hepatocellular Carcinoma. Gastroenterology (2019) 156(2):477-91.el. doi: 10.1053/j.gastro. 2018.08.065

4. Dhanasekaran R, Nault JC, Roberts LR, Zucman-Rossi J. Genomic Medicine and Implications for Hepatocellular Carcinoma Prevention and Therapy. Gastroenterology (2019) 156(2):492-509. doi: 10.1053/j.gastro.2018.11.001

5. Zhou M, Zhang Q, Zhao J, Liao M, Wen S, Yang M. Phosphorylation of Bcl-2 Plays an Important Role in Glycochenodeoxycholate-Induced Survival and Chemoresistance in HCC. Oncol Rep (2017) 38(3):1742-50. doi: 10.3892/ or.2017.5830

6. Jaiswal K, Lopez-Guzman C, Souza RF, Spechler SJ, Sarosi GAJr. Bile Salt Exposure Increases Proliferation Through P38 and ERK MAPK Pathways in a Non-Neoplastic Barrett's Cell Line. Am J Physiol Gastrointest Liver Physiol (2006) 290(2):G335-42. doi: 10.1152/ajpgi.00167.2005

7. Jaiswal K, Tello V, Lopez-Guzman C, Nwariaku F, Anthony T, Sarosi GAJr. Bile Salt Exposure Causes Phosphatidyl-Inositol-3-Kinase-Mediated Proliferation in a Barrett's Adenocarcinoma Cell Line. Surgery (2004) 136 (2):160-8. doi: 10.1016/j.surg.2004.04.008

8. Ishizuka S, Shiwaku M, Hagio M, Suzuki T, Hira T, Hara H. Glycochenodeoxycholic Acid Promotes Proliferation of Intestinal Epithelia via Reduction of Cyclic AMP and Increase in H2AX Phosphorylation After Exposure to $\gamma$-Rays. Biomed Res (2012) 33(3)159-65. doi: 10.2220/biomedres.33.159

9. Komichi D, Tazuma S, Nishioka T, Hyogo H, Chayama K. Glycochenodeoxycholate Plays a Carcinogenic Role in Immortalized Mouse Cholangiocytes via Oxidative DNA Damage. Free Radic Biol Med (2005) 39 (11):1418-27. doi: 10.1016/j.freeradbiomed.2005.07.005

10. Wang C, Yang M, Zhao J, Li X, Xiao X, Zhang Y, et al. Bile Salt (Glycochenodeoxycholate Acid) Induces Cell Survival and Chemoresistance in Hepatocellular Carcinoma. J Cell Physiol (2019) 234(7):10899-906. doi: $10.1002 /$ jcp. 27905

11. Sun MH, Chen XC, Han M, Yang YN, Gao XM, Ma X, et al. Cardioprotective Effects of Constitutively Active MEK1 Against H2O2-Induced Apoptosis and Autophagy in Cardiomyocytes via the ERK1/2 Signaling Pathway. Biochem Biophys Res Commun (2019) 512(1):125-30. doi: 10.1016/j.bbrc.2019.03.008

12. Caragarajah BJ, Khokhlatchev A, Cobb MH, Goldsmith EJ. Activation Mechanism of the MAP Kinase ERK2 by Dual Phosphorylation. Cell (1997) 90(5):859-69. doi: 10.1016/S0092-8674(00)80351-7

13. Yao Z, Seger R. The ERK Signaling Cascade-Views From Different Subcellular Compartments. BioFactors (Oxford England) (2009) 35(5):407-16. doi: 10.1002/biof.52

14. Cook SJ, Stuart K, Gilley R, Sale MJ. Control of Cell Death and Mitochondrial Fission by ERK1/2 MAP Kinase Signalling. FEBS J (2017) 284(24):4177-95. doi: $10.1111 /$ febs. 14122

15. Wainstein E, Seger R. The Dynamic Subcellular Localization of ERK: Mechanisms of Translocation and Role. Curr Opin Cell Biol (2016) 39:1520. doi: 10.1016/j.ceb.2016.01.007

16. Sale MJ, Cook SJ. That Which Does Not Kill Me Makes Me Stronger; Combining ERK1/2 Pathway. Br J Pharmacol (2013) 169(8):1708-22. and the Natural Science Foundation of Hunan Province (grant numbers 2020JJ4889, 2018JJ3830, and 2016JJ3177).

\section{SUPPLEMENTARY MATERIAL}

The Supplementary Material for this article can be found online at: https://www.frontiersin.org/articles/10.3389/fonc.2021. 663944/full\#supplementary-material

17. Xie B, Zhang L, Hu W, Fan M, Jiang N, Duan Y, et al. Dual Blockage of STAT3 and ERK1/2 Eliminates Radioresistant GBM Cells. Redox Biol (2019) 24:101189. doi: 10.1016/j.redox.2019.101189

18. Hsieh MJ, Wang CW, Lin JT, Chuang YC, Hsi YT, Lo YS, et al. Celastrol, a Plant-Derived Triterpene, Induces Cisplatin-Resistance Nasopharyngeal Carcinoma Cancer Cell Apoptosis Though ERK1/2 and P38 MAPK Signaling Pathway. Phytomed Int J Phytotherapy Phytopharmacol (2019) 58:152805. doi: 10.1016/j.phymed.2018.12.028

19. Zhong J, Yu X, Dong X, Lu H, Zhou W, Li L, et al. Downregulation of Secreted Clusterin Potentiates the Lethality of Sorafenib in Hepatocellular Carcinoma in Association With the Inhibition of ERK1/2 Signals. Int J Mol Med (2018) 41 (5):2893-900. doi: 10.3892/ijmm.2018.3463

20. Liao M, Zhao J, Wang T, Duan J, Zhang Y, Deng X. Role of Bile Salt in Regulating Mcl-1 Phosphorylation and Chemoresistance in Hepatocellular Carcinoma Cells. Mol Cancer (2011) 10:44. doi: 10.1186/1476-4598-10-44

21. Chen M, Zhuang C, Liu Y, Li J, Dai F, Xia M, et al. Tetracycline-Inducible shRNA Targeting Antisense Long Non-Coding RNA HIF1A-AS2 Represses the Malignant Phenotypes of Bladder Cancer. Cancer Lett (2016) 376(1):15564. doi: 10.1016/j.canlet.2016.03.037

22. Chen M, Li J, Zhuang C, Cai Z. Increased lncRNA ABHD11-AS1 Represses the Malignant Phenotypes of Bladder Cancer. Oncotarget (2017) 8(17):2817686. doi: 10.18632/oncotarget14945

23. Chen M, Zhang R, Lu L, Du J, Chen C, Ding K, et al. LncRNA PVT1 Accelerates Malignant Phenotypes of Bladder Cancer Cells by Modulating. Aging (Albany NY) (2020) 12(21):22291-312. doi: 10.18632/aging.202203

24. Ghosh S. Cisplatin: The First Metal Based Anticancer Drug. Bioorg Chem (2019) 88:102925. doi: 10.1016/j.bioorg.2019.102925

25. Sun Z, Niu S, Xu F, Zhao W, Ma R, Chen M. CircAMOTL1 Promotes Tumorigenesis Through miR-526b/SIK2 Axis in Cervical Cancer. Front Cell Dev Biol (2020) 8:568190. doi: 10.3389/fcell.2020.568190

26. Marin JJG, Cives-Losada C, Asensio M, Lozano E, Briz O, Macias RIR. Mechanisms of Anticancer Drug Resistance in Hepatoblastoma. Cancers (2019) 11(3). doi: 10.3390/cancers11030407

27. Chen LC, Shibu MA, Liu CJ, Han CK, Ju DT, Chen PY, et al. ERK1/2 Mediates the Lipopolysaccharide-Induced Upregulation of FGF-2, uPA, MMP-2, MMP-9 and Cellular Migration in Cardiac Fibroblasts. Chemico-biol Interact (2019) 306:62-9. doi: 10.1016/j.cbi.2019.04.010

28. Wang K, Brems JJ, Gamelli RL, Ding J. Reversibility of Caspase Activation and its Role During Glycochenodeoxycholate-Induced Hepatocyte Apoptosis. J Biol Chem (2005) 280(25):23490-5. doi: 10.1074/jbc.M411607200

29. Gao L, Lv G, Li R, Liu WT, Zong C, Ye F, et al. Glycochenodeoxycholate Promotes Hepatocellular Carcinoma Invasion and Migration by AMPK/ mTOR Dependent Autophagy Activation. Cancer Lett (2019) 454:215-23. doi: 10.1016/j.canlet.2019.04.009

30. Odagiri N, Matsubara T, Higuchi M, Takada S, Urushima H, Sato-Matsubara $\mathrm{M}$, et al. Involvement of ERK1/2 Activation in the Gene Expression of Senescence-Associated Secretory Factors in Human Hepatic Stellate Cells. Mol Cell Biochem (2019) 455(1-2):7-19. doi: 10.1007/s11010-018-3466-x

31. Li H, Zhang M, Linghu E, Zhou F, Herman JG, Hu L, et al. Epigenetic Silencing of TMEM176A Activates ERK Signaling in Human Hepatocellular Carcinoma. Clin Epigenet (2018) 10(1):137. doi: 10.1186/s13148-018-0570-4

32. Hou H, Ge C, Sun H, Li H, Li J, Tian H. Tunicamycin Inhibits Cell Proliferation and Migration in Hepatocellular Carcinoma Through Suppression of CD44s and the ERK1/2 Pathway. Cancer Sci (2018) 109 (4):1088-100. doi: $10.1111 /$ cas.13518 
33. Chen P, Lei L, Wang J, Zou X, Zhang D, Deng L, et al. Downregulation of Talin1 Promotes Hepatocellular Carcinoma Progression Through Activation of the ERK1/2 Pathway. Cancer Sci (2017) 108(6):1157-68. doi: 10.1111/ cas. 13247

34. Cong N, Li Z, Shao W, Li J, Yu S. Activation of ETA Receptor by Endothelin-1 Induces Hepatocellular Carcinoma Cell Migration and Invasion via ERK1/2 and AKT Signaling Pathway. J Membrane Biol (2016) 249(1-2):119-28. doi: 10.1007/s00232-015-9854-1

35. Wei Y, Zhang X, Wen S, Huang S, Huang Q, Lu S, et al. Methyl Helicterate Inhibits Hepatic Stellate Cell Activation Through Downregulating the ERK1/2 Signaling Pathway. J Cell Biochem (2019). doi: 10.1002/jcb.28756

36. Raška J, Čtveráčková L, Dydowiczová A, Sovadinová I, Bláha L, Babica P. Cylindrospermopsin Induces Cellular Stress and Activation of ERK1/2 and P38 MAPK Pathways in Adult Human Liver Stem Cells. Chemosphere (2019) 227:43-52. doi: 10.1016/j.chemosphere.2019.03.131

37. Xie YX, Liao R, Pan L, Du CY. ERK Pathway Activation Contributes to the Tumor-Promoting Effects of Hepatic Stellate Cells in Hepatocellular Carcinoma. Immunol Lett (2017) 188:116-23. doi: 10.1016/j.imlet.2017.06.009

38. Wortzel I, Seger R. The ERK Cascade: Distinct Functions Within Various Subcellular Organelle. Genes Cancer (2011) 2(3):195-209. doi: 10.1177/ 1947601911407328

39. Liu YL, Lai F, Wilmott JS, Yan XG, Liu XY, Luan Q, et al. Noxa Upregulation by Oncogenic Activation of MEK/ERK Through CREB Promotes. Oncotarget (2014) 5(22):11237-51. doi: 10.18632/oncotarget.2616

40. Domina AM, Vrana JA, Gregory MA, Hann SR, Craig RW. MCL1 is Phosphorylated in the PEST Region and Stabilized Upon ERK Activation in
Viable Cells, and at Additional sites With Cytotoxic Okadaic Acid or Taxol. Oncogene (2004) 23(31):5301-15.

41. Maurer U, Charvet C, Wagman AS, Dejardin E, Green DR. Glycogen Synthase Kinase-3 Regulates Mitochondrial Outer Membrane. Mol Cell (2006) 21 (6):749-60.

42. Yang JY, Zong CS, Xia W, Yamaguchi H, Ding Q, Xie X, et al. ERK Promotes Tumorigenesis by Inhibiting FOXO3a via MDM2-Mediated Degradation. Nat Cell Biol (2008) 10(2):138-48.doi: 10.1038/ncb1676

43. Ley R, Balmanno K, Hadfield K, Weston C, Cook SJ. Activation of the ERK1/2 Signaling Pathway Promotes Phosphorylation and Proteasome-Dependent Degradation of the BH3-Only Protein, Bim. J Biol Chem (2003) 278 (21):18811-6.

44. Kuwana T, Hayes LB, Chipuk JE, Bonzon C, Sullivan BA, Green DR, et al. BH3 Domains of BH3-Only Proteins Differentially Regulate Bax-Mediated. Mol Cell (2005) 17(4):525-35. doi: 10.1016/jmolcel200502003

Conflict of Interest: The authors declare that the research was conducted in the absence of any commercial or financial relationships that could be construed as a potential conflict of interest.

Copyright (c) $2021 \mathrm{Li}$, Zhou, Wang, Xu and Yang. This is an open-access article distributed under the terms of the Creative Commons Attribution License (CC BY). The use, distribution or reproduction in other forums is permitted, provided the original author(s) and the copyright owner(s) are credited and that the original publication in this journal is cited, in accordance with accepted academic practice. No use, distribution or reproduction is permitted which does not comply with these terms. 